\title{
EL DERECHO A LA SALUD UNA BÚSQUEDA INACABADA PARA LA SOCIEDAD COLOMBIANA
}

Wilson Giovanni Jiménez Barbosa*

\section{RESUMEN}

La salud a pesar de no haber sido definida como un Derecho Fundamental en la Constitución Nacional de 1991, si debe ser entendida como tal, gracias a la interpretación jurisprudencial hecha por la Corte Constitucional en varias de sus sentencias, según las cuales el goce de plena salud se halla conexo con el derecho a la vida y a la dignidad humana.

Sin embargo, desde la implementación de la Ley 100 de 1993, la vulneración a este derecho ha sido reiterativo, por parte de los diferentes actores que hacen parte del Sistema General de Seguridad Social en Salud, lo que ha impulsado a los usuarios a defender su derecho mediante

la interposición de acciones de tutela,

lo que ha generado congestión en los despachos judiciales.

\section{Palabras clave}

Sentencia

Corte Constitucional

Tutela

Comité técnico cientifico

Planes de beneficios

Ministerio de la Protección Social

Entidad Promotora de Salud-EPS

\section{InTRODUCCIón}

Ua reforma al Sistema $\mathrm{Na}$ cional de Salud, realizada en desarrollo de los contenidos de los Artículos 48 y 49 de la Constitución Política Colombiana en el año de 1993, contenida en el Libro Segundo de la Ley 100, convirtió la salud en Colombia en un bien transable, generador de lucro para unos pocos particulares a costa de la salud y la vida de los colombianos.

Este sistema, se basó en un modelo de aseguramiento, respondiendo así a criterios dados por organismos de financiamiento multilaterales, tales como el Banco Mundial, el Fondo Monetario Internacional y el Banco Interamericano de Desarrollo, los cuales impulsaron

* Odontólogo Universidad Nacional de Colombia, especialista en Gerencia de Servicios de Salud y en Auditoría de la Calidad en Salud, Universidad Jorge Tadeo Lozano. Magister en Administración Universidad de La Salle. Aspirante a doctor en Ciencias Sociales, Niñez y Juventud, Universidad de Manizales. 
la implementación de los lineamientos condensados en el denominado consenso de Washington.

Como resultado, el modelo colombiano se ha centrado en resultados económicos y no en resultados de salud, lo que ha llevado al aumento de la carga de la enfermedad y a la negación del acceso a los servicios, lo que ha generado una constante vulneración al derecho a la salud.

Para demostrar lo anterior, a continuación se pasará a describir sucintamente la historia de la seguridad social en salud en Colombia, para continuar con la descripción del uso de la acción constitucional de amparo de tutela en defensa del Derecho a la Salud, prosiguiendo con las acciones realizadas por la Corte Constitucional a través de sus sentencias, en la búsqueda de su protección y finalizando con un análisis de las reacciones del Gobierno Nacional ante estas.

\section{ABSTRACT}

As health has not just been defined as a fundamental right in the 1991 National Constitution, if it is understood as it is, thanks to the jurisprudential interpretation made by the Constitutional Court in some of its statements, according to which the right to health is linked with the Right to Live and the Human Dignity.

Since the implementation of the 100 Law in 1993, the violation of this right has been permanently seen, in the hands of the different agents who are part of the Health Social Security System, which has made all its clients defend their rights through the use of guardianship, generating a lot of burocratic delay in judicial offices.

Additionally, some of the guardianships belong to the category of unjustified negotiations made by insurance services which are part of the portfolio offered in the Obligatory Health Plan and which are also part of the existing inequity in this system, caused by the non fulfillment of its universal coverage and its content differences between the benefits granted by the contributive and subsided current plans.

To end these problems in the SGSSS and to guarantee the right to health of all citizens, the Constitutional Court passed the C-463 and T- 760 statements in 2008, which have turned into guidelines for the different agents who intervene in this system, determining how to conduct real actions.

Finally, the interpretation made by the National Government on these issues have caused a lot of doubts on the question if the defined objectives will be reached or this will become just another failure in an attempt to provide the right to health.

\section{KEY WORDS}

Statement, Constitutional Court, Guardianship, Scientific and Technical Committee, Benefit plans, Ministry of Health and Social Security, EPS. 


\section{RESEÑa HISTÓRICA DE LA SEGURIDAD SOCIAL Y EL SISTEMA DE SALUD EN COLOMBIA}

\section{Los conceptos y estructuras} propios de la seguridad social han sido introducidos tardíamente en Colombia y siempre en forma reactiva a procesos históricos externos e internos. En este sentido se puede mencionar como momentos fundamentales en este proceso de construcción de la seguridad social en Colombia, la promulgación en 1915 -bajo el gobierno de José Vicente Concha- de la Ley de Indemnizaciones por accidentes de trabajo, como un primer acercamiento a la implementación del modelo alemán de seguridad social, el cual para ese momento ya llevaba más de treinta años de haberse desarrollado en Europa.

Sin embargo, la consolidación de este modelo solo se alcanzaría mediante la creación del Instituto Colombiano de los Seguros Sociales - ICSS en 1946, nombre que posteriormente sería modificado a Instituto del Seguro Social ISS, cuya financiación debía ser tripartita entre gobierno, patronos y trabajadores, con una cobertura en servicios limitada al trabajador, dejando desprotegida a la familia del mismo.

Dicha deficiencia en la cobertura familiar, se trató de compensar mediante la creación de las cajas de previsión social y las cajas de compensación familiar, en el período comprendido entre 1948 a 1950, las primeras como privilegio para los trabajadores y las familias de empresas públicas en cuanto a acceso a servicios de salud y las segundas, en una primera instancia como una forma redistributiva del ingreso entre la totalidad de los trabajadores, expresado en bonos económicos para cada hijo de los trabajadores de menores ingresos; posteriormente esta figura evolucionó a brindar servicios de salud, educativos, financieros, de vivienda y en los últimos años, subsidios de desempleo, a todos sus afiliados, constituyéndolas en una de las más sólidas experiencias en seguridad social del país.

Pero el modelo así constituido era fragmentario y excluyente, en razón a que para alcanzar los beneficios se debía estar inmerso en el círculo económico formal, mediante una relación laboral, lo cual tampoco se constituía en condición de facto, porque los patronos buscaban evadir la afiliación al ISS para reducir los costos laborales. Es así, que para 1993 la cobertura del aseguramiento era del $18 \%$ del total de la población nacional y sólo cubría al $50 \%$ de los trabajadores asalariados, Barón (2006).

Como se deduce, el modelo dejaba desprotegida de aseguramiento al $82 \%$ de la población nacional, la cual, en el caso de la más pobre, tenía que recurrir a los servicios públicos de salud para dar 
solución a sus necesidades, pero estos eran desarticulados, insuficientes y su acceso y distribución geográfica estaba ligado altamente a intereses de índole político. Como resultado, para 1993 en el caso de salud la cobertura del aseguramiento sólo alcanzaba al 18\% de la pobla-ción nacional, Ministerio de Salud (1994).

El avance histórico conllevó que como resultado de procesos políticos internos, se promulgara el 4 de Julio de 1991 una nueva constitución nacional, que en su artículo primero expresó que Colombia sería un Estado Social de Derecho, lo que constituyó un gran avance en la garantía de una seguridad social universal, equitativa y solidaria, bajo un enfoque de garantía de los derechos fundamentales a todo habitante del territorio nacional. Sin embargo, este avance se produjo en un momento de grandes transformaciones globales en los órdenes social, económico y político, que repercutieron en la orientación filosófica de la Constitución Nacional y en las reformas que se desprendieron de ella.

Dentro de estas, es de gran importancia para el caso colombiano, el denominado consenso de Washington, difundido en 1989 como producto de análisis adelantados por académicos y economistas norteamericanos, funcionarios de gobierno de ese país y funcionarios del Banco Mundial y el Fondo Monetario Internacional, el cual estableció un decálogo de políticas a ser implementadas en primera instancia en Latinoamérica, pero que posteriormente fueron implantadas en la mayor parte del mundo, las cuales fueron:

- Establecer una disciplina fiscal.

- Priorizar el gasto público en educación y salud.

- Llevar a cabo una reforma tributaria.

- Establecer tasas de interés positivas determinadas por el mercado.

- Lograr tipos de cambio competitivos.

- Desarrollar políticas comerciales liberales.

- Una mayor apertura a la inversión extranjera.

- Privatizar las empresas públicas.

- Llevar a cabo una profunda desregulación

- Garantizar la protección de la propiedad privada.

Como se evidencia, el consenso de Washington profesa la reducción del gasto público en servicios sociales tales como salud, educación, vivienda y trabajo, para permitir a los gobiernos de los países receptores de créditos lograr equilibrios fiscales que les posibilite el cumplimiento de los pagos a la deuda, en detrimento de las condiciones de calidad de vida de sus poblaciones. Es así como aparece en el país el paradigma 
neoliberal de la eficiencia del mercado como garante de la prestación de todos los servicios sociales que antes brindaba el Estado, reduciendo la función de este a una esfera netamente reguladora.

Bajo la actual crisis global, los preceptos del consenso están siendo revaluados en los países centrales que encabezan la banca multilateral, sin embargo en Colombia aún se sigue planeando la política social bajo este anacrónico esquema neoliberal.

Bajo este contexto, empiezan a promulgarse Leyes que desarrollan esta nueva orientación del Estado, dentro de las cuales es relevante la Ley 100 de 1993 la cual creó el denominado "Sistema de Seguridad Social Integral", compuesta centralmente por cuatro libros, el primero referente a pensiones, el segundo a salud, un tercero para riesgos profesionales y el libro cuarto a servicios sociales complementarios.

Este modelo, se basa en el denominado pluralismo estructurado, concepto desarrollado por Juan Luís Londoño y Julio Frenk (1997), el cual introduce la figura de la agencia como un intermediario entre el prestador de un servicio y su receptor, como forma de compensar la vulnerabilidad de este último frente a un monopolio público o la asimetría de la información -en el caso de un prestador privado-, buscando distribuir y equilibrar el poder entre los diferentes actores.
Para el caso colombiano, con dicho fin en el sector salud se implementaron varios cambios entre los que se destacan: la eliminación del monopolio público de la seguridad social, abriéndose la libre competencia mediante la creación de las denominadas Entidades Promotoras de Salud -EPS-; la constitución de entes reguladores con participación social cono el Consejo Nacional de Seguridad Social; la transformación del modelo de financiamiento del sistema pasando de uno basado en subsidios, a la oferta a uno soportado en subsidios a la demanda apoyado en un sistema financiero con componente solidario a través del Fondo de Solidaridad y Garantía -FOSYGA y la constitución de dos regímenes uno orientado a la inclusión de la población con capacidad de pago denominado Contributivo y otro cuya función es incorporar a la población pobre y vulnerable, según focalización establecida de acuerdo a las necesidades básicas insatisfechas de los núcleos familiares, realizada a través del instrumento diseñado por el Departamento Nacional de Planeación, llamado Sistema de Información de Beneficiarios para Programas Sociales -SISBEN-, denominado Subsidiado; la ampliación de la red de prestadores de salud especialmente privados, la exigencia de autofinanciamiento de la red pública mediante su transformación en entes autónomos descentralizados denominados Empresas Sociales del estado - ESE, entre otras. 
Sin embargo, dada la imposibilidad inicial de lograr cobertura universal por parte de los dos regímenes establecidos, la Ley 100 planteó una figura transitoria para la población, que siendo pobre y vulnerable no pudiese ser afiliada al régimen subsidiado, y que se denominó vinculada.

El derecho a la salud de esta población -debería ser en teoríamaterializado por el Estado a través de los fondos locales de salud de los diferentes entes territoriales; pero en la práctica el derecho a la salud le es vulnerado dada la incapacidad financiera de los Departamentos y Municipios para brindar su cobertura.

En lo referente a los planes de beneficios, la incapacidad financiera inicial del Sistema General de Seguridad Social en Salud, se vio reflejada en la diferenciación de los contenidos del POS contributivo y del POS subsidiado, en detrimento deesteúltimo en una diferencia que si se evalúa porcentualmente con base en los valores de las Unidades de Pago por Capitación, que corresponden al dinero que reciben las I EPS tanto de los regímenes contributivo y subsidiado por cada uno de sus afiliados con el fin de garantizar la prestación del POS, de cada uno de los regímenes, su relación es de 1:56.

El legislador en 1993, consciente de estas falencia estableció como plazo perentorio el 31 de diciembre del año 2000, para que el SGSSS alcanzara la cobertura universal del aseguramiento con equidad en los contenidos de los planes de beneficios de ambos regímenes, sin embargo, esta meta fue incumplida y a la fecha no ha sido alcanzada.

Como se puede observar, el modelo es netamente economicista, dejando de lado y sólo como un resultado secundario, el impacto que el mismo pueda producir sobre la salud pública del país, lo que ha llevado a un deterioro de la misma, reflejado en el descenso de indicadores como el de vacunación, el cual se redujo en forma lineal entre 1993 y 1999, Rojas, Zapata, Alzate y Rodríguez (2008); el aumento de la incidencia de enfermedades de transmisión sexual en poblaciones de riesgo tales como jóvenes y amas de casa y el aumento de la incidencia de patologías como dengue, leishmaniasis y fiebre amarilla, además del incremento en el número de casos de patologías crónicas degenerativas prevenibles tales como enfermedad renal y diabetes mellitus.

\section{Lo anterior se debe a factores} tales como, la orientación económico asistencialista y poco preventiva que las EPS contemplan dentro de sus modelos de atención, la atomización de los recursos destinados a la promoción y la prevención entre gobierno nacional, departamental, distrital y municipal, EPS del régimen contributivo y subsidiado y Administradoras de Riesgos Profesionales -ARP-, además de la poca participación social en la planeación, gestión y control del sistema, lo que lo ha convertido en ajeno a los contextos 
donde opera especialmente en las regiones apartadas del país y en poblaciones específicas como juventudes, comunidades indígenas y negras, pueblos rom, desplazados, trabajadores de la economía informal, mujeres cabeza de familia, donde y en quienes las lógicas del mercado no operan y/o sus problemáticas no se ven reflejadas dentro del sistema.

Pero en contraste, las políticas nacionales que buscaron corregir estas falencias, no se construyeron en procesos participativos amplios, que incluyeran las particularidades de cada una de las regiones y poblaciones del país, sino que respondieron a una nueva generación de lineamientos dados desde los centros del poder, en este caso a través del Banco Mundial, mediante el documento elaborado por Holzmann y Jorgensen (2000) titulado Manejo Social del Riesgo: un nuevo marco conceptual para protección social y más allá, según el cual, La idea revolucionaria que define la frontera entre la era moderna y el pasado es el dominio del riesgo: la noción de que el futuro es más que un capricho divino y que los hombres y mujeres no son inermes frente a la naturaleza.

De acuerdo con este marco, para el Banco Mundial la protección social consta de dos grandes componentes: el primero orientado a brindar intervenciones públicas para asistir a los individuos, hogares y comunidades, con el fin de que estos aprendan a manejar y controlar el riesgo de forma adecuada; el segundo componente, se encamina a ofrecer apoyo a personas en situación crítica, definidas éstas últimas como los pobres que no se pueden mantener a sí mismos, incluso si existieran oportunidades de empleo, pues quedan excluidos de la asignación de recursos realizada por el mercado.

Esta categoría, denominada población en situación crítica, da soporte a la adopción de políticas de focalización, mediante las cuales los potenciales beneficiarios de programas sociales financiados con subsidios estatales, deben demostrar su condición de alta vulnerabilidad, para ser objeto del apoyo requerido.

Es en este contexto, las comunidades, gremios y academia, manifestaron los diferentes análisis sobre las necesidades en salud, en los diversos foros que se suscitaron en todo el país, lo que llevó a que se adelantará un debate al interior del congreso colombiano con el fin de reformar el Libro Segundo de la Ley 100 de 1993; proceso que se realizó en un lapso de tres años, con no pocos tropiezos, dados los fuertes intereses económicos y políticos en juego, en especial manifestados por las EPS, lo cuales impidieron una reforma estructural que satisficiera las expectativas de los diferentes estamentos.

Por el contrario, la reforma promulgada en la Ley 1122 de 2007, en varios de sus contenidos es regresiva para la participación ciudadana, como es el caso de la conformación de la Comisión de Regulación en Salud, la 
cual se apropia de las funciones fundamentales que tenía el Consejo Nacional de Seguridad Social, convirtiendo al principal órgano de dirección y regulación del sistema de un espacio de concertación con los sectores sociales a un organismo técnico con nula participación social.

En igual sentido, la reforma renuncia en forma tacita a la aspiración de cobertura universal en salud, limitándola a las poblaciones con capacidad de pago afiliadas al Régimen Contributivo y a las poblaciones focalizadas por el SISBEN en los niveles 1, 2 y 3, lo que deja por fuera a amplios sectores de las capas medias de la sociedad, especialmente los ligados a la economía informal, a los que adicionalmente se les dificultó su ingreso al Contributivo al aumentárseles la cotización del $12 \%$ al $12.5 \%$ de su ingreso base, lo que sumado a lo establecido en la Ley 797 de 2003, mediante la cual se debe hacer aportes similares y obligatorios a pensiones, generó una problemática social al quedar excluidos de la seguridad social sectores, en especial jóvenes, quienes al ingresar a la fuerza laboral son contratados en condiciones de desfavorabilidad, dadas las políticas de flexibilidad laboral y tercerización para la contención de costos en la producción.

$Y$ en lo referente a los planes de beneficios, lejos de saldar la inequidad existente, ésta fue agravada en la reforma contenida en la Ley 1122 al desarrollar los denominados subsidios parciales, los cuales tienen contenidos en servicios inferiores a los del ya limitado POS subsidiado, estableciendo una relación con el plan de beneficios del Régimen Contributivo, de acuerdo a sus respectivas Unidades de Pago por Capitación de 1:22.

3. La tutella como garante DEL DERECHO DE SALUD

Como resultado de las inequidades que el SGSSS ha mantenido a lo largo de los años de su desarrollo e implementación, en lo referente a coberturas poblacionales y de contenidos de los planes de beneficios, se facilitó que las EPS tanto del Régimen Contributivo como del Subsidiado, hayan procedido a hacer negaciones injustificadas de servicios contenidos en el Plan Obligatorio de Salud - POS y en forma negligente no operar con diligencia los Comités Técnico Científicos - CTC que permitieran la autorización de los servicios no POS sin recurrir al amparo de tutela.

Inconvenientes aun más complejos, han enfrentado las poblaciones denominadas vinculadas al exigir el acceso a los servicios de salud en los hospitales públicos, ya que a éstos se les han venido reduciendo paulatinamente los recursos que les son transferidos, cuya destinación es la atención con cargo al subsidio a la oferta, lo que 
les limita su capacidad receptora de pacientes. Lo anterior sumado a las trabas que encuentra este tipo usuarios en las secretarías de salud territoriales, para la autorización de servicios de mediana y alta complejidad, ha llevado a que este grupo de ciudadanos les sea violentado con mayor facilidad su derecho a la salud.

Lo anterior, dio como resultado que el único camino viable que encontraron los usuarios del sistema para lograr la garantía plena de su derecho a la salud haya sido la interposición de acciones de tutela, generando congestión judicial.

Lo anterior encuentra soporte en los hallazgos hechos por la Defensoría del Pueblo (2007) en su estudio "La Tutela y el Derecho a la Salud: período 2003 -2005", en el cual se expresa cómo más de un tercio de las acciones de tutela interpuestas en el país corresponden a vulneración al derecho a la salud, de las cuales el $87 \%$ son resueltas a favor del accionante y de las denegadas un buen número corresponde a muerte de quien la interpuso o a hechos superados al haberse subsanado la violación por parte de la entidad aseguradora.

Pero causa una gran preocupación, que el estudio encuentre que el $77 \%$ de las tutelas son interpuestas contra las EPS de ambos regímenes y el $14.6 \%$ contra los entes territoriales. Al analizar estos resultados por regímenes, es más bajo el indicador por cada 10.000 afiliados para el Régimen Subsidiado, lo que podría estará asociado a que en éste existe un margen máximo de gasto administrativo, lo cual no ocurre en el Régimen Contributivo, en el que el menor gasto en salud le implica mayores utilidades económicas a las EPS.

Lo anterior se agrava, si se tiene en cuenta que el $56.4 \%$ de las acciones de tutela interpuestas contra las entidades del aseguramiento, corresponden a negaciones de los contenidos del POS, lo que es facilitado por los vacios normativos en cuanto a sus contenidos expresados en la Resolución 5261 de 1994.

Otro factor evidente en el estudio, es la visión cortoplacista de las EPS, las cuales niegan el acceso a actividades de orden preventivo tales como citologías vaginales, mamografías y antígenos prostáticos, así como a citas con especialidades de las diferentes áreas del sector salud, que evitarían en un futuro que el SGSSS tuviera que asumir costos elevados derivados del tratamiento de patologías que pudieron ser evitadas o diagnosticadas en fases tempranas de su evolución, además evitar el sufrimiento emocional al paciente y su familia. 


\section{4.}

\section{LAS ACCIONES EMPRENDIDAS POR LA CORTE CONSTITUCIONAL PARA GARANTIZAR EL DERECHO A LA SALUD A TODOS LOS COLOMBIANOS}

Bajo este panorama, la Corte Constitucional profirió en el año 2008 las Sentencias C-463 y T-760, con el fin de restablecer metas y dinámicas incumplidas tanto por el Gobierno Nacional como por las EPS. En tal sentido, la Corte estableció en las sentencias, entre otros, los siguientes objetivos:

- Aprobar o rechazar la prestación de servicios, actividades, procedimientos o intervenciones no incluidos dentro del POS a través de los comités Técnico Científicos, so pena de que si por no haberse surtido este proceso se interpone una acción de tutela para garantizar su prestación, la EPS deberá responder con el $50 \%$ del valor de las acciones tuteladas.

- Actualizar integralmente los Planes Obligatorios de Salud (POS) los cuales deberán adoptarse antes de 1 de febrero de 2009.

- Actualizar los Planes Obligatorios de Salud por lo menos una vez al año.

- Garantizar que todas las EPS realicen informes que permitan evidenciar los motivos causantes de la negación de servicios.
- Identificar las EPS y las IPS que con mayor frecuencia se niegan a autorizar oportunamente los servicios de salud incluidos en el POS.

- Ordenar a la Comisión de Regulación en Salud unificar los planes de beneficios para los niños y las niñas del régimen contributivo y del subsidiado, medida que deberá adoptarse antes del 1 de octubre de 2009.

- Ordenar a la Comisión de Regulación en Salud que adopte un programa y un cronograma para la unificación gradual y sostenible de los planes de beneficios del régimen contributivo y del régimen subsidiado.

- Ordenar al Ministerio de la Protección Social y al administrador fiduciario del FOSYGA que adopten medidas para garantizar que el procedimiento de recobro por parte de las EPS ante el FOSYGA, así como el de los entes territoriales, sea ágil y asegure el flujo oportuno y suficiente de los recursos.

- Ordenar al Ministerio de Protección Social que adopte las medidas necesarias para asegurar la cobertura universal sostenible del SGSSS, antes de enero de 2010. 
Como se deduce, muchos de los objetivos planteados en la Sentencias C-463 y T-760, buscan encaminar al SGSSS hacia el alcance de los principios constitucionales de universalidad, solidaridad, igualdad y eficiencia, estableciendo plazos perentorios para el logro de las metas incumplidas.

Las otras medidas son conducentes a la búsqueda de procesos eficientes que permitan al interior del
SGSSS la existencia de una dinámica tanto en la prestación de los servicios como de su financiación.

La necesidad que tuvo la Corte Constitucional de proferir estas sentencias refleja la inoperancia del Gobierno Nacional a lo largo de estos quince años para regular, controlar, inspeccionar y evaluar el SGSSS, lo que ha permitido, la conculcación sistemática del derecho a la salud de los colombianos.

\section{5.}

HAN TENIDO EN LOS DIFERENTES ACTORES DEL SGSSS

Arente a los planteamientos hechos por la corte en sus sentencias, con preocupación se observa como el Ministerio de la Protección Social en lugar de tomar caminos claros hacia el cumplimiento de estos objetivos, ha empezado a presentar objeciones desde el punto de vista económico, al expresar el Jefe de esta cartera que para dar cumplimiento a lo establecido en la sentencia se requieren 6 billones de pesos y que según él no están disponibles en el presupuesto nacional.

Lo que el Ministro no menciona es que el Gobierno Nacional le debe a la subcuenta de solidaridad del FOSYGA, porlos períodos comprendidos entre 1994 a 2006, cerca de 2 billones de pesos por concepto de los recursos que acorde a la Ley 100 de 1993, debían ser aportados del presupuesto nacional en relación de $1: 1$ con los aportes que de la cotización que pagan los afiliados al Régimen Contributivo son transferidos a ésta subcuenta, lo que se conoce como el parypassu, Esmeral y Niño (2006) y que invertidos en TES y en CDTs se hayan cerca de 4 billones de pesos de excedentes de las diferentes subcuentas del FOSYGA, es decir, que el incumplimiento de las metas es causado por la negligente aplicación de los recursos que le deberían corresponder al Sistema, los cuales se han invertido en pro de equilibrios 
fiscales y rendimientos financieros y en detrimento de la salud de los colombianos.

Adicionalmente, las anteriores cifras no tienen en cuenta, que las investigaciones realizadas por Giraldo y Martínez (2008) y plasmadas en su artículo "Impacto para la sociedad y el estado del no pago de la porción social del salario por actividad económica", demuestran que debido a la flexibilización laboral impulsada por el gobierno nacional, se ha generado una cultura de no pago de aportes en salud por parte de patronos, mediante figuras de contratación como la de prestación de servicios, representa un costo cercano a los 2 billones de pesos que el estado le está subsidiando al sector empresarial.

En lo referente a la equiparación de los contenidos de los planes de beneficios de los regímenes, es preocupante la posibilidad planteada por el Ministerio de la Protección Social de reformar dichos contenidos pasando de una orientación centrada en actividades y procedimientos a una enfocada en patologías, ya que la misma puede convertirse en una medida regresiva que reduce los reales contenidos del POS igualándolo por lo bajo, situación que sólo beneficia a las EPS, quienes verían reducido su gasto en salud y por ende elevando sus utilidades por vía legislativa y no como resultado del mejoramiento de los estados de salud de sus poblaciones.

Adicionalmente, esta regresión generaría mayor presión sobre el FOSYGA quien tendría que responder financieramente por un mayor número de Comités Técnico Científicos o de tutelas.

Por tanto, debe hacérsele un control político desde ya a esta posible redefinición de los contenidos del POS como garantía del derecho a la salud de los colombianos.

De otra parte, los resultados financieros de las EPS en los últimos años, de acuerdo con lo publicado por la revista Semana (2009), reflejan baja o nula rentabilidad operacional; afirmación, que si se revisa los contenidos del libro de Gilberto Barón (2007) "Las Cuentas de Salud de Colombia 1993-2003" no resulta cierta, ya que en un ítem llamado "otros usos", las EPS se quedan con el $30 \%$ de los recursos que deberían garantizar la prestación del POS a sus afiliados.

Esto se refuerza, si se tiene en cuenta que al revisar las cifras de este libro, se puede establecer, que antes de la Ley 100 de 1993 de cada $\$ 100$ pesos que existían en el sistema $\$ 88$ se gastaban en servicios de salud y que actualmente sólo se gastan $\$ 65$.

Pero además, si se aceptaran los resultados, estos serían explicables por las erráticas políticas de promoción y prevención que estos empresarios cortoplacistas de la salud han implementado a lo largo de estos quince años.

En conclusión, el modelo es tan falente que posiblemente en el 
mediano plazo ni siquiera será un buen negocio, dado que las EPS habrán elevado la carga de la enfermedad, habiéndose llevado el lucro en la primera etapa del modelo y dejándole el coste final al estado, quien tendrá que elevar el gasto destinado al sector salud, para poder dar una respuesta oportuna a las necesidades de la población colombiana.

La hipótesis emergente de estos planteamientos es que es necesario derogar el actual modelo y establecer uno nuevo que no se centre en el lucro sino en la obtención de resultados positivos para la salud de las poblaciones, para ello es necesario el adoptar un sistema orientado a garantizar la universalidad en la cobertura, basado en una visión de salud pública integral que garantice el acceso oportuno a los programas de promoción y prevención de la enfermedad, así como a su diagnóstico, tratamiento y rehabilitación, es decir que permita disfrutar a todos los colombianos de un estado de bienestar pleno, gracias a la plena garantía de su Derecho a la Salud.

\section{Bibliografía}

Barón L, G. (2007). Cuentas de Salud de Colombia 1993 - 2003. Ministerio de la Protección Social, Programa de Apoyo a la Reforma.

Bushnell, David. (1994). Colombia una nación a pesar de sí misma. Editorial Planeta.

Castaño, Ramón A. (2005) ¿Estado, mercado y análisis económico: complementos, o substitutos?. Pontificia Universidad Javeriana, Revista Gerencia y Políticas de salud, número 8.

Constitución Política de Colombia, 1991.

Corte Constitucional Colombiana. Sentencia C-463

Corte Constitucional Colombiana. Sentencia T-760.

Emmerich, G. y Alarcón, V. (2007). Tratado de Ciencia Política. Editorial Anthropos.

Esmeral, M.y Niño V. (2006). Análisis del Cumplimiento de los Principios Constitucionales de la Seguridad Social Durante la Implementación del Libro Segundo de la Ley 100 de 1993, Periodo 1993 - 2005. Trabajo de Grado, Programa de Optometría, Universidad de La Salle. 
Fresneda Bautista, O. (diciembre 2003). La Focalización en el Régimen Subsidiado de Salud: Elementos para un Balnce. Pontificia Universidad Javeriana, Revista Gerencia y Políticas de salud, número 5.

Giraldo, C. y Martínez, F. (2008) "Impacto para la sociedad y el estado del no pago de la porción social del salario por actividad económica". Revista CIFE, Universidad Santo Tomás, año 10, número 13, páginas 125 a la 150.

Holzmann, R. y Jørgensen, O. Manejo social del riesgo: un nuevo marco conceptual para la protección social y más allá. Revista Facultad Nacional de Salud Pública 2003; 21(1): 73-106.

Jaramillo Pérez, I. Tendencias en la reorganización de los sistemas de salud.

Londoño, J. y Frenk, J. Pluralismo Estructurado: Hacia un modelo innovador para la reforma de los sistemas de salud en América Latina. Banco Interamericano de Desarrollo Oficina del Economista Jefe, Documento de Trabajo 353.

Ministerio de Salud. (1994). La reforma a la seguridad social en salud. Tomo 1: Antecedentes y resultados. Santafé de Bogotá.

Salazar Silva, F. La Configuración del Estado de Bienestar, Elementos Constitutivos.

Presidencia de la República de Colombia. Ley 100 de 1993 Sistema de Seguridad Social Integral.

Presidencia de la República de Colombia. Ley 1122 de 2007. Por la cual se hacen algunas modificaciones en el Sistema General de Seguridad Social en Salud y se dictan otras disposiciones.

Profamilia. Encuesta Nacional de Demografía y Salud. Año 2005.

Revista Semana. Las 100 Empresas más grandes de Colombia. Publicaciones Semana, Mayo de 2009.

Rojas, J. et.al (2008): Cobertura vacunal en nños de 12 a 47 meses de edad en estratos 1 y 2 de la Zona Urbana de Cali, año 2002. Colombia Médica, Versión Online, ISSN 1657-9534. Universidad del Valle. 